\title{
Thermal inertias in the upper millimeters of the Martian surface derived using Phobos' shadow
}

\author{
Bruce H. Betts, ${ }^{1}$ Bruce C. Murray, and Tomáx Svítek ${ }^{2}$ \\ Division of Geological and Planetary Sciences, Califormia Institute of Technology, Pasadena
}

\begin{abstract}
The first thermal images of Phobos' shadow on the surface of Mars, in addition to simultaneous visible images, were obtained by the Phobos ' 88 Termoskan instrument. The best observed shadow occurrence was on the flanks of Arsia Mons. For this occurrence, we combined the observed decrease in visible illumination of the surface with the observed decrease in brightness temperature to calculate thermal inertias of the Martian surface. The most realistic of our three models of eclipse cooling improves upon our preliminary model by including nonisothermal initial conditions and downward atmospheric flux. Most of our derived inertias fall within the range 38 to $59 \mathrm{~J} \mathrm{~m}^{-2} \mathrm{~s}^{-1 / 2} \mathrm{~K}^{-1}\left(0.9\right.$ to $\left.1.410^{-3} \mathrm{cal} \mathrm{cm}^{-2} \mathrm{~s}^{-1 / 2} \mathrm{~K}^{-1}\right)$, corresponding to dust-sized particles (for a homogeneous surface), consistent with previous theories of Tharsis as a current area of dust deposition. Viking infrared thermal mapper (IRTM) inertias are diurnally derived and are sensitive to centimeter depths, whereas the shadow-derived inertias sample the upper tenths of a millimeter of the surface. The shadowderived inertias are lower than those derived from Viking IRTM measurements (84 to 147), however, uncertainties in both sets of derived inertias make conclusions about layering tenuous. Thus, near-surface millimeter versus centimeter layering may exist in this region, but if it does, it is likely not very significant. Both eclipse and diurnal inertias appear to increase near the eastern end of the shadow occurrence. We also analyzed a shadow occurrence near the crater Herschel that showed no observed cooling. This analysis was limited by cool morning temperatures and instrument sensitivity, but yielded a lower bound of 80 on eclipse inertias in that region. Based upon our results, we strongly recommend future spacecraft thermal observations of Phobos' shadow, and suggest that they will be most useful if they improve upon Termoskan's geographic and temporal coverage and its accuracy.
\end{abstract}

\section{Introduction}

Mars' moon Phobos orbits Mars in a roughly 8-hour, circular, equatorial orbit at an altitude of approximately $6000 \mathrm{~km}$. During the time periods surrounding the Martian equinoxes, Phobos casts a completely penumbral shadow on the surface of Mars' equatorial regions during portions of each orbit. A passing of the shadow would be viewed by an observer on the surface as a partial eclipse lasting roughly $20 \mathrm{~s}$. The Termoskan instrument on board the Soviet Phobos '88 spacecraft obtained the first thermal images of Phobos' shadow on the surface of Mars. Simultaneous visible images were also obtained.

Termoskan was an optical-mechanical scanning radiometer with one visible channel $(0.5-1.0 \mu \mathrm{m})$ and one thermal infrared channel (8.5-12.0 $\mu \mathrm{m}$ ) (see Selivanov et al. [1989], Murray et al. [1991], and Betts [1993] for more information). The instrument was fixed to the spacecraft, pointing in the antisolar direction. Termoskan used a scanning mirror to build up the north-south component of image panoramas and the spacecraft's motion to build the east-west component.

\footnotetext{
INow at the San Juan Capistrano Research Institute, San Juan Capistrano, California.

${ }^{2}$ Now at Orbital Sciences Corporation, Dulles, Virginia.
}

Copyright 1995 by the American Geophysical Union.

Paper number 95JE00226.

0148-0227/95/95JE-00226\$05.00
Termoskan observed the shadow of Phobos on the surface of Mars during two of its four panoramas. Using these observations, we have been able to combine the observed decrease in visible illumination of the surface with the observed decrease in brightness temperature to calculate thermal inertias of the uppermost tenths of a millimeter of the Martian surface. Thermal inertia, a bulk measure of the resistance of a unit surface area to changes in temperature, is commonly used to characterize the insulating properties of planetary surfaces. It is defined as $l=\left(k p c_{p}\right)^{1 / 2}$, where $k$ is the thermal conductivity, $\rho$ is the bulk density, and $c_{p}$ is the specific heat of the material. Lowinertia materials have smaller thermal skin depths and heat and cool more quickly than high inertia materials. In this paper, thermal inertia values are given in SI units $\left(\mathrm{J} \mathrm{m}^{-2} \mathrm{~K}^{-1} \mathrm{~S}^{-1 / 2}\right)$ with inertia values in the units $\left(10^{-3} \mathrm{cal} \mathrm{cm}^{-2} \mathrm{~K}^{-1} \mathrm{~S}^{-1 / 2}\right)$ often used for the Martian surface [e.g., Kieffer et al., 1977] given in parentheses following the SI values.

Murray et al. [1991] presented a summary of the preliminary results of an analysis of the best observed and least complicated occurrence of the shadow (near Arsia Mars; see Figure 1). We now present for the first time (1) a refined analysis of that shadow occurrence that incorporates two additional model factors: nonisothermal start conditions and atmospheric reradiation, and that includes inertias as a function of longitude in the shadowed region; (2) an analysis of another shadow occurrence which was observed near the crater Herschel; (3) a detailed description of the models used; (4) a comparison with the diumal inertias of Hayashi et al. [this issue], which are based upon the combined surface atmosphere (CSA) model of Haberle 

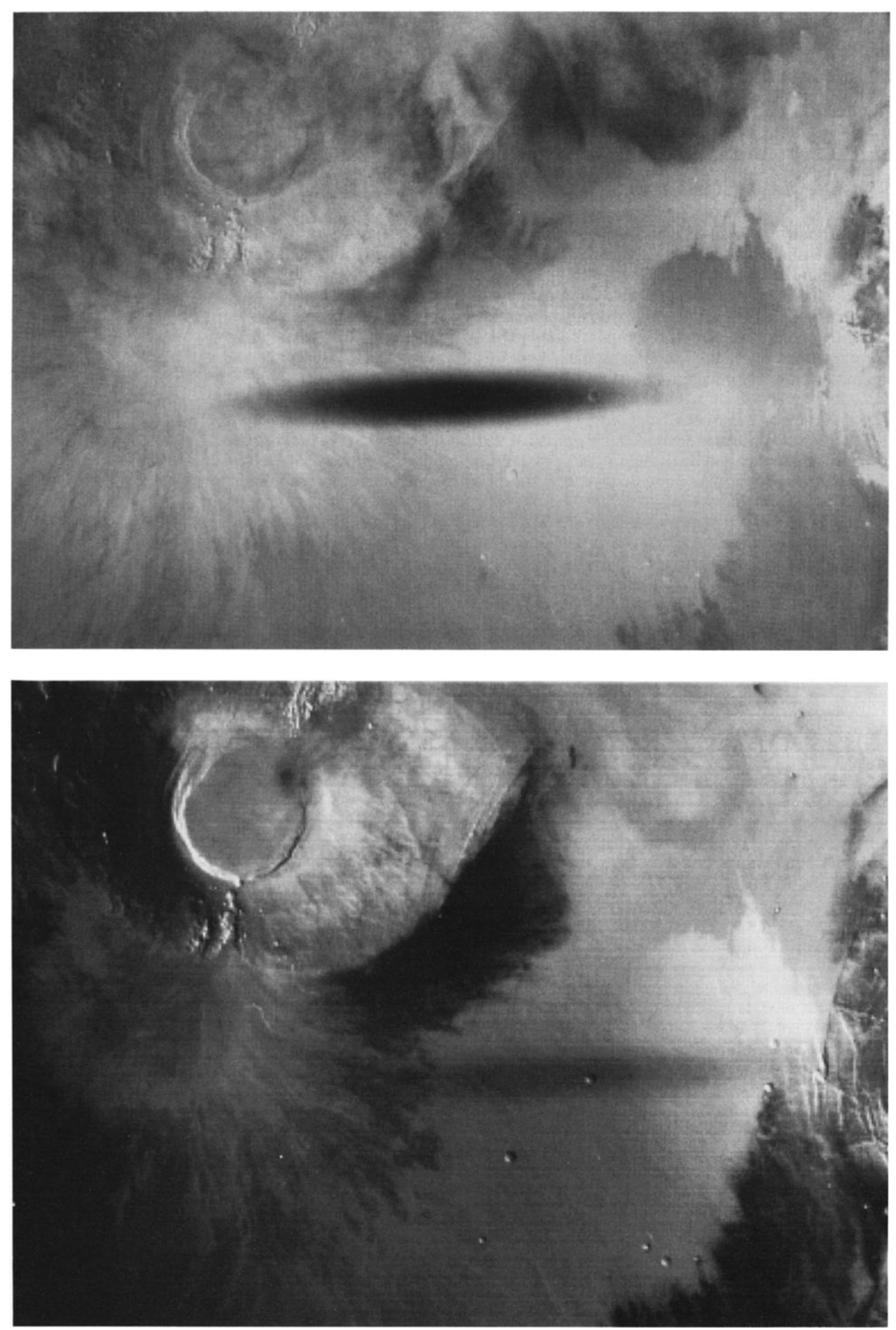
Table 1. Phobos Shadow Occurrences Within the Termoskan Data

\begin{tabular}{|c|c|c|c|c|c|}
\hline Occurrence & Scan & Location & $\begin{array}{c}\text { West } \\
\text { Longitudes } \\
\text { Covered, deg }\end{array}$ & $\begin{array}{l}\text { Times of Day } \\
\text { Covered. } \\
\text { hours }\end{array}$ & Notes \\
\hline 1 & 3 & Flanks of Arsia Mons & 110 to 120 & 9.3 to 10.1 & Analyzed here. \\
\hline 2 & 3 & $\begin{array}{l}\text { S. of western half of } \\
\text { Valles Marineris }\end{array}$ & 75 to 105 & 10.5 to 12.7 & \\
\hline 3 & 4 & $\begin{array}{l}\text { Eastern end is in the } \\
\text { crater Herschel }\end{array}$ & 228 to 255 & 7.4 to 9.5 & $\begin{array}{l}\text { No cooling apparent in thermal data. } \\
\text { Analyzed here. }\end{array}$ \\
\hline 4 & 4 & $\begin{array}{l}\text { North of Ma'adim } \\
\text { Vallis }\end{array}$ & 172 to 210 & 10.8 to 13.8 & $\begin{array}{l}\text { No visible channel data for eastern end } \\
\text { of shadow. Dropped lines in visible } \\
\text { data that do exist. }\end{array}$ \\
\hline
\end{tabular}

All shadow occurrences are centered approximately upon $14^{\circ} \mathrm{S}$ latitude.

and Jokosky [1991]; and (5) implications for the planning and analysis of future similar observations.

Section 2 of this paper gives an overview of the observations and the thermal models we used. Section 3 compares the Termoskan thermal data with the model results to derive thermal inertias. Section 4 discusses the results, and Section 5 discusses and the potential for future Phobos shadow research. The appendix describes in detail the three thermal models, including the inputs that were used from the Termoskan data.

\section{Overview of Observations and Modeling}

The elongated shape of the Phobos shadow (Figure 1) in the Termoskan panoramas was due to a fortuitous combination of the scanning nature of the instrument, unusual orbital geometry (the spacecraft and Phobos were nearly co-orbiting), the antisolar orientation of the instrument, and a slight rocking of the spacecraft. The similarity of the spacecraft's orbit to Phobos' orbit combined with Termoskan's antisolar orientation (zero solar phase angle) conspired to put Termoskan's instantaneous field of view near the location of the Phobos shadow as it traveled across Mars' surface. However, because the spacecraft and moon were not actually in the same place, this orientation alone would have missed observing the shadow. "Fortunately," the spacecraft, and hence Termoskan, rocked slightly back and forth. Thus, Termoskan's instantaneous field of view rocked into and out of observing the shadow (see Murray et al. [1991] or Betts [1993] for more detailed descriptions of the observations).

Termoskan's field of view rocked into observing Phobos' shadow four times, twice in each of two observing sessions, both on March 26, 1989. The four shadow occurrences, their locations, and their basic differences are summarized in Table 1. All are centered roughly on $14^{\circ} \mathrm{S}$ latitude.

Unfortunately, in terms of ease of analysis, each of the shadow occurrences has different characteristics. The Arsia Mons occurrence (number 1 in Table 1) was the best observed and is able to yield the most information. Hence, it is the focus of our modeling and analysis presented here.

We also present an analysis of occurrence 3 (the "Herschel occurrence"); however, that analysis is necessarily simplified and much less accurate because no cooling from the passage of the shadow was detected. Thus, only a lower bound on thermal inertia could be determined by making assumptions about what level of cooling could be detected in the Termoskan data.

Occurrences 2 and 4 are both more complex than the Arsia occurrence. The shadows themselves appear longer (cover a wider range of longitudes), and the E-W shadow profiles do not appear as smooth and symmetric (smoothly darkening, then brightening) as the Arsia shadow profile. These two factors imply that the spacecraft rocking motion was slower and that the assumption of uniform rocking motion (used in our analysis and discussed in the appendix) is less likely to be valid. In addition, the greater apparent lengths of the shadows imply that a wider range of local times of day, surrounding temperatures, and albedos were observed. Occurrence 4 additionally lacks complete visible data coverage.

For the Arsia and Herschel occurrences, we used the observed drop in the visible flux within the shadowed area to model the solar insolation as a function of both actual time since the beginning of eclipse and position in the scan. We then used this in three different one-dimensional, finite difference thermal models for homogeneous surfaces (adapted from Clifford et al. [1987]). Model 1 was our preliminary model [Betts et al., 1990; Murnay et al., 1991]. Model 2 does not assume that the preeclipse temperatures are constant with depth as Model 1 did. Instead it uses a temperature with depth profile derived from a diumal thermal model (as discussed in the appendix). Haberle and Jakosky [1991] concluded that atmospheric effects are less important for eclipse-derived thermal inertias than for diurnally derived thermal inertias, based upon theoretical considerations

Figure 1. Phobos shadow images. (Top) Termoskan visible and (Bottom) thermal images showing the Phobos shadow occurrence on the flanks of Arsia Mons. North is towards the top. Contrast has been enhanced to emphasize the shadow. The elongated shape of the shadow is due to a fortuitous combination of the scanning nature of the instrument, unusual orbital geometry (the spacecraft and Phobos were nearly co-orbiting), the antisolar orientation of the instrument, and a slight rocking motion of the spacecraft. Note that the shadow is observed first (i.e., further west because the instrument built up images from west to east) in the visible, then later (to the east) in the thermal. This is due to the delay in cooling after the onset of the shadow. 
involving effects of the short duration of the eclipse relative to a diumal period, and the relatively high daytime temperatures (which cause surface emission to dominate atmospheric emission). In order to test this and to ascertain the magnitude of atmospheric effects, we created model 3 by adding a downward atmospheric flux term to model 2. Model 3 is the most realistic of the three models, but due to the uncertainty in the downward atmospheric flux, it is useful to consider model 2 separately. For the Arsia occurrence, we present the results of all three models here for comparison and completeness (see the appendix for detailed descriptions of all three models.)

\section{Comparison of Data With Models}

\subsection{Arsia Occurrence}

To best compare Termoskan eclipse temperatures with model results, we used temperature drops within the eclipse rather than absolute temperatures. Variations in absolute temperature are observed outside the eclipse due to variations in albedo, inertia, and time of day. These factors also will affect the observed temperatures within the eclipse. To minimize these effects, we did two things to the data used for comparison. First, for points within and outside the shadow, we averaged 10-pixel (in the east-west direction) by 1-pixel strips. Second, the data we compare with models are estimated temperature drops within the eclipse. We find these temperature drops by averaging the temperatures of points to either side (north and south) of the shadow and then subtracting the temperature observed in the middle of the shadow. Using these temperature drops rather than absolute temperatures is particularly effective at removing time of day effects. Also, because the area happens to be reasonably bland thermally, gradual albedo and diumal thermal inertia variations are also removed. In addition, by using temperature drops, we reduce the effects of atmospheric scattering, which should act approximately equally inside and outside the shadow.

To plot the data versus the model curves, these temperature drops were subtracted from $255 \mathrm{~K}$, the pre-eclipse temperature used in the models. Figure 2 shows the data plotted versus model 1 curves. Figure 3 shows an analogous plot for model 2, Figure 4 shows curves for model 3 with a downward atmospheric flux $\left(A_{f}\right)$ of $20 \%$ of the absorbed pre-eclipse solar flux, and Figure 5 shows curves for model 3 with $A_{f}=10 \%$. Data error bars are discussed in section 4.2.1., and although they are of interest in interpreting physical results, they do not affect comparisons of different models. Most of the data fall between inertias of $38(0.9)$ and $50(1.2)$ for model 1, between $41(1.0)$ and 67 (1.6) for model 2, between $35(0.8)$ and $50(1.2)$ for model 3 with $A_{f}=20 \%$, and between $38(0.9)$ and $59(1.4)$ for model 3 with $A_{f}=10 \%$. In all cases, inertias are slightly higher later (towards the east) in the shadowed region.

\subsection{Herschel Occurrence}

For the Herschel occurrence the shadow appears clearly in Termoskan's visible channel, but cooling from the shadow does

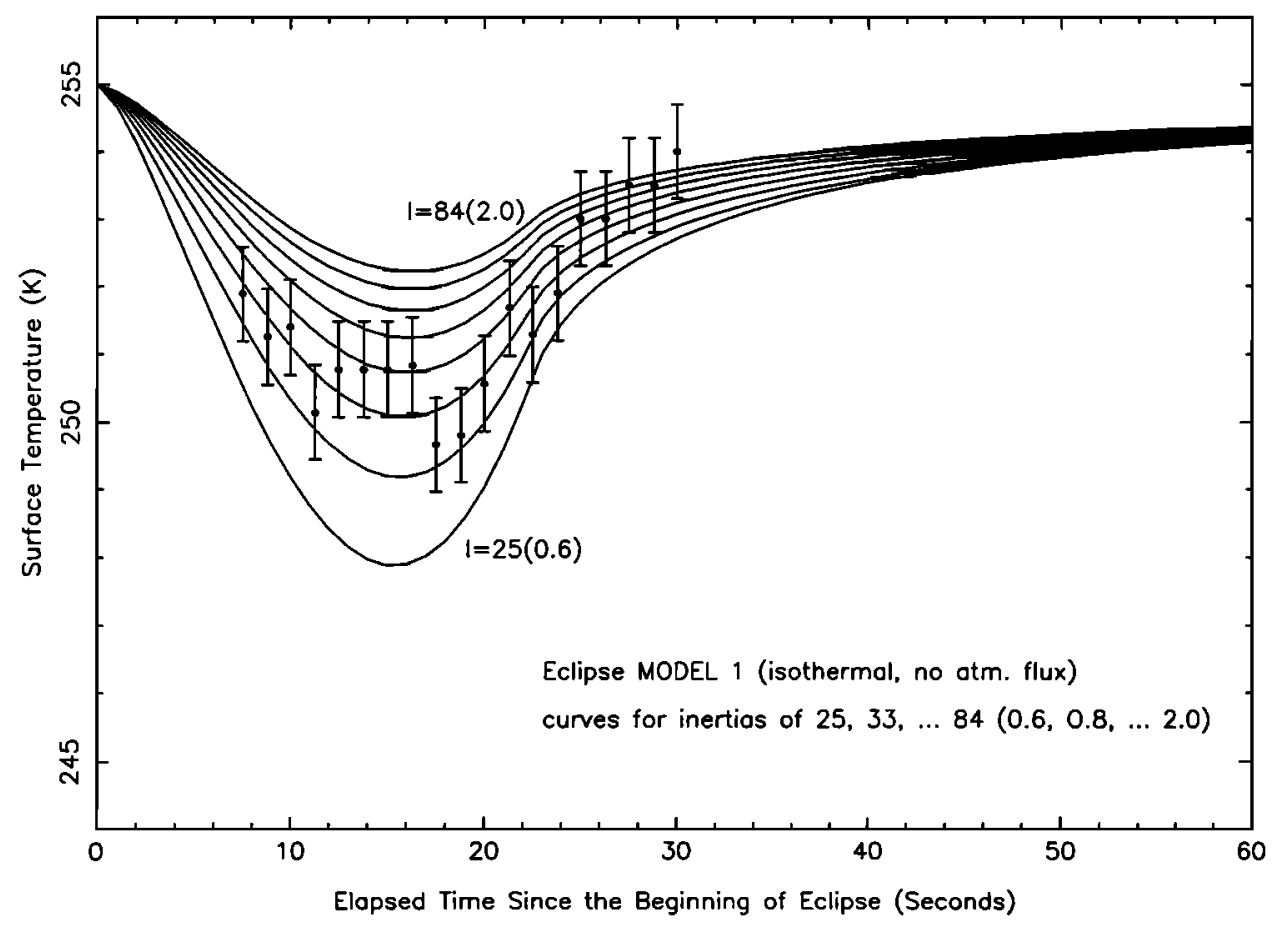

Figure 2. Eclipse model 1 results. The lines in this plot are model surface temperatures for various thermal inertias from 25 to $84 \mathrm{~J} \mathrm{~m}^{-2} \mathrm{~s}^{-1 / 2} \mathrm{~K}^{-1}\left(0.6\right.$ to 2.0 in units of $\left.10^{-3} \mathrm{cal} \mathrm{cm}^{-2} \mathrm{~K}^{-1} \mathrm{~s}^{-1 / 2}\right)$. The dots are observational data retrieved from the Termoskan thermal infrared channel for the shadow occurrence near Arsia Mons. They are derived from 10-line averages of temperature drops at the center of the shadow relative to comparable points outside the shadow. Size of the error bars is discussed in the text. The temperature drops have been subtracted from $255 \mathrm{~K}$ to facilitate comparison with our model results. We chose $255 \mathrm{~K}$ for the model because it is a typical temperature from the region surrounding the shadow. Most of the observational data values fall between model curves corresponding to thermal inertias of $38(0.9)$ to $50(1.2)$. Results in this figure are the same as those presented by Murroy et al. [1991]. 


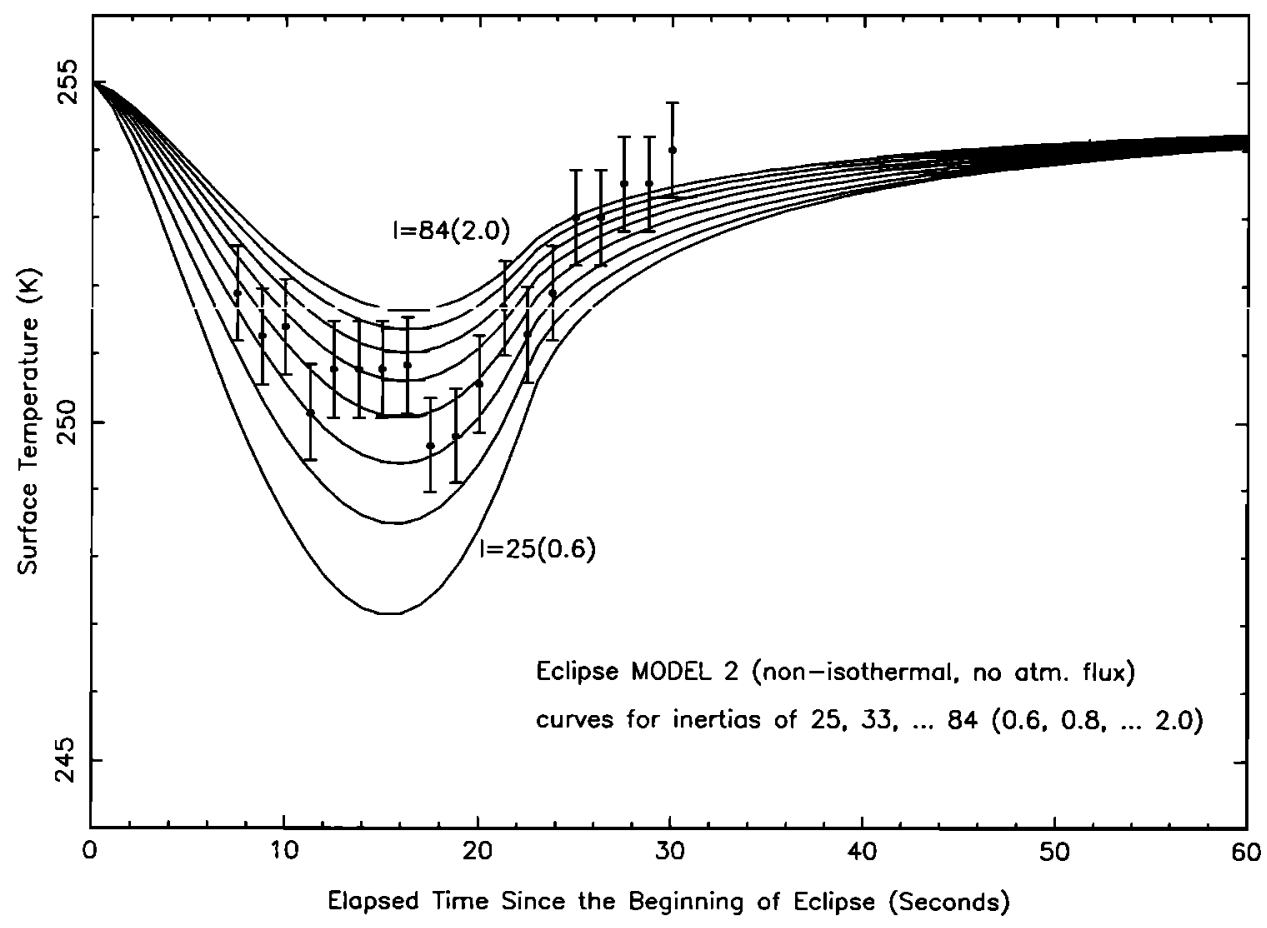

Figure 3. Eclipse model 2 results. Same as Figure 2 but for model 2 results. Model 2 added nonisothermal with depth pre-eclipse conditions to model 1. Most of the observational data values fall between model curves corresponding to thermal inertias of 41 (1.0) to $67(1.6)$.

not show up in the thermal channel. Thus, for this occurrence, lower bound on eclipse inertias for the region near the center of we cannot measure temperature drops. However, we can the shadow. Modeling was carried out as before but with the estimate the level of cooling that could have been detected by appropriate changes in time of day, insolation, albedo, and Termoskan, and compare that value to model results to derive a starting temperatures. We only ran one of our models: Model 3

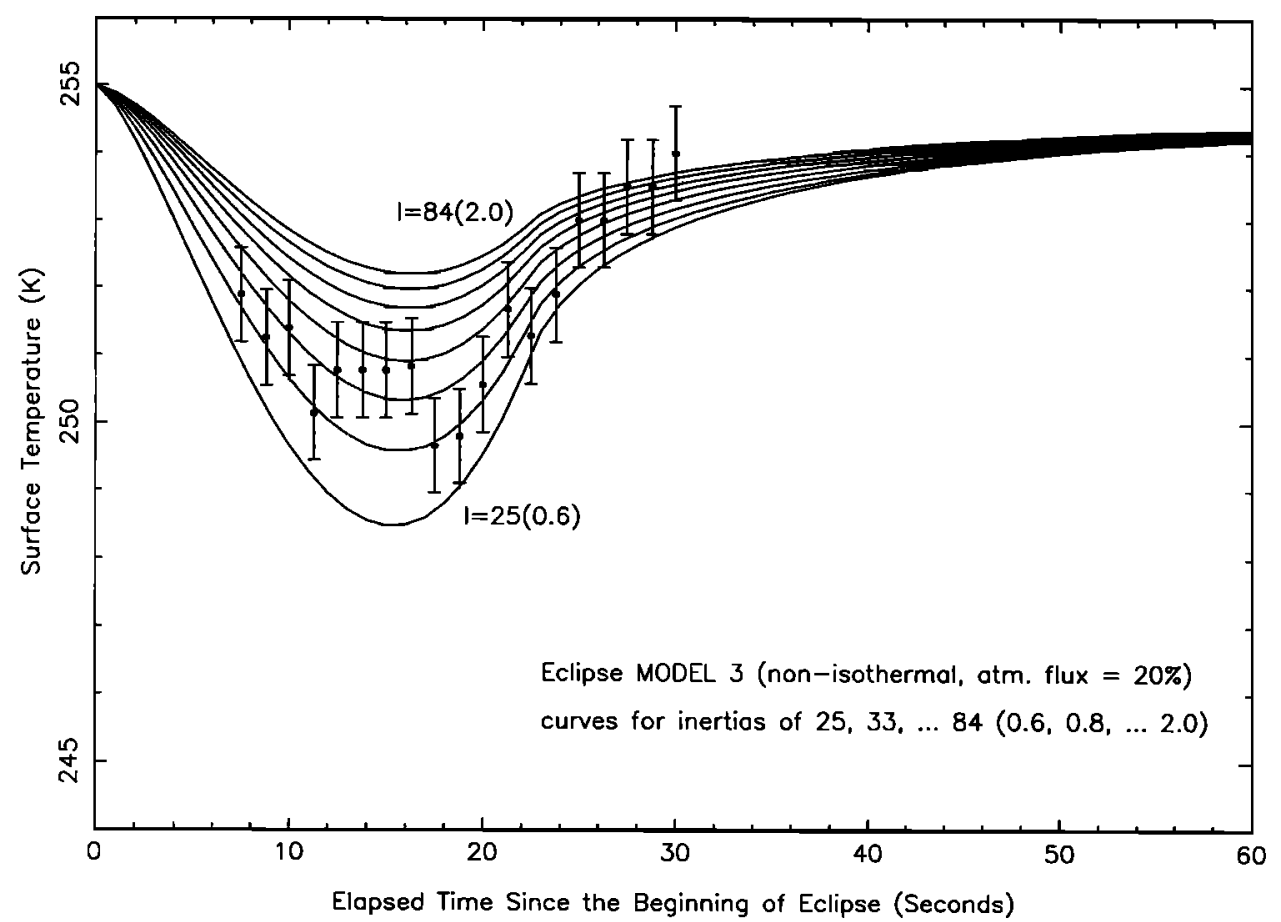

Figure 4. Eclipse model 3, $A_{f}=20 \%$. Same as Figure 3 but for model 3 results. Model 3 added atmospheric downward flux to model 2 . The curves plotted here are for a downward atmospheric flux of $20 \%$ of the absorbed pre-eclipse solar flux. Most of the observational data values fall between model curves corresponding to thermal inertias of $35(0.8)$ to $50(1.2)$. 


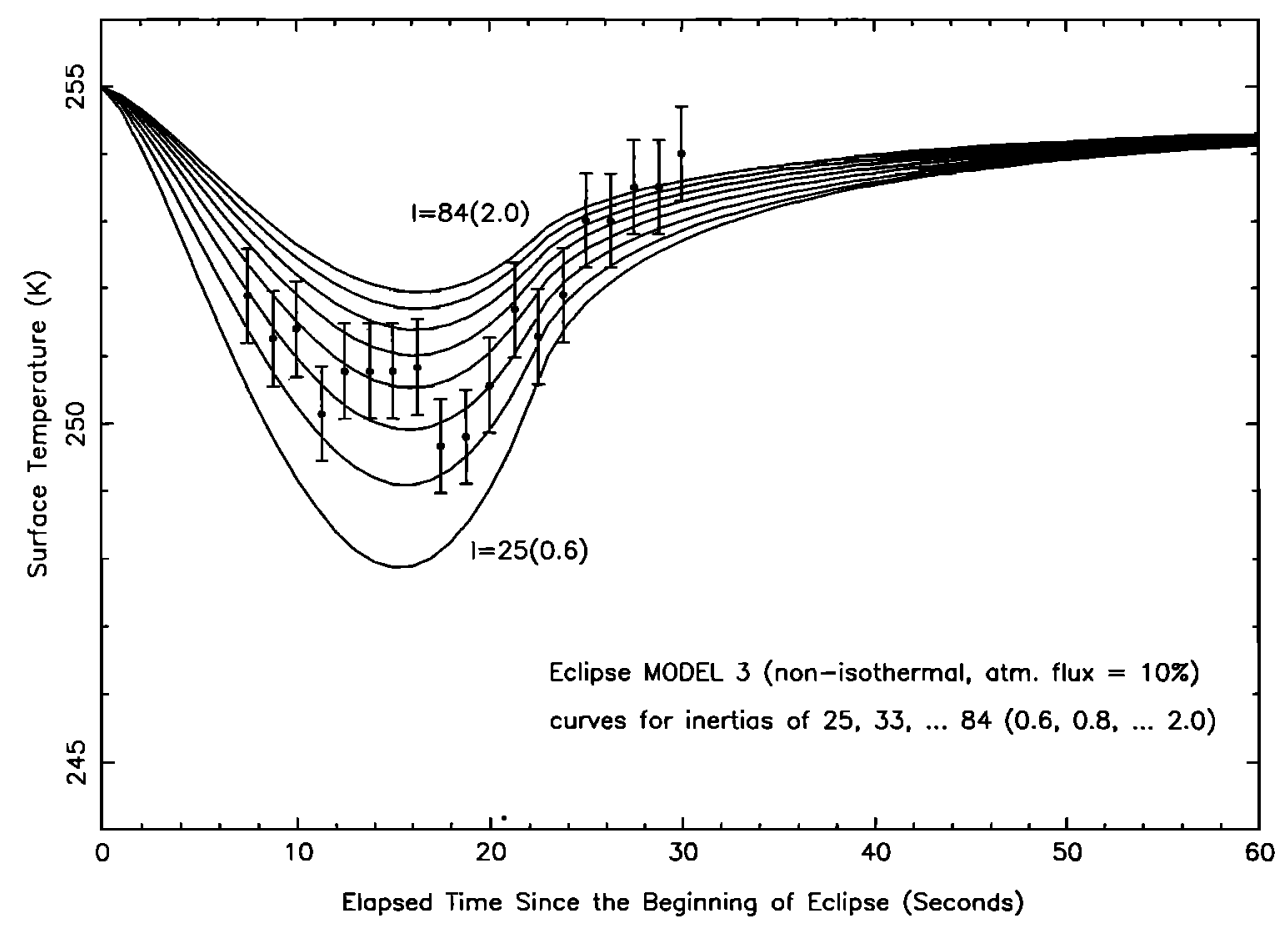

Figure 5. Eclipse model 3, $A_{f}=10 \%$. Same as Figure 4 but with $10 \%$ downward atmospheric flux. Most of the observational data values fall between model curves corresponding to thermal inertias of $38(0.9)$ to 59 (1.4). We consider this the most realistic model of those presented for the Arsia occurrence.

with $A_{f}=10 \%$, which we determined to be our most realistic model (discussed below). We compared the maximum amount of modeled cooling for several thermal inertias with an estimate of the amount of cooling that could have been detected in the Termoskan data either by image processing or digital data analysis.

We estimate for this occurrence that we could have detected shadow cooling corresponding to a drop in the infrared channel of approximately $3 \mathrm{DN}$ (data numbers) relative to the surrounding unshadowed area. This is based upon tested image processing of the data, comparisons with other occurrences of the shadow, and attempts to detect simulated shadow cooling effects. Infrared channel variations in this region due to inherent terrain variations are also on the order of 2 or $3 \mathrm{DN}$, but we are aided in looking for shadow cooling by the image nature of the data and the fact that we know where the shadow should be based upon the visible channel data.

For the temperatures in the Herschel region, 3 DN corresponds to approximately $2.5 \mathrm{~K}$. Comparing with our model results, we find that a $2.5 \mathrm{~K}$ temperature drop corresponds to an eclipse inertia of approximately 80 (2). Thus, the data provide an approximate lower bound of 80 (2) for the thermal inertia in the upper millimeter of the region near the center of the shadow (approximately $14^{\circ} \mathrm{S}, 241^{\circ} \mathrm{W}$ ). At first, it may seem counterintuitive that no observed cooling would only constrain the inertia to be above a value as low as 80 (2). After all, the very obvious cooling observed in the Arsia occurrence corresponds to inertias near 40 (1). However, the following factors make more accurate constraint impossible: (1) The Herschel occurrence was much cooler than the Arsia occurrence due primarily to the earlier moming observations as well as the inherently higher diumal inertias in the Herschel region. Termoskan had less sensitivity at cooler temperatures (see
Murray et al. [1991] or Betts [1993] for the infrared response characteristics), e.g., $3 \mathrm{DN}$ at $230 \mathrm{~K}$ correspond to about $2.5 \mathrm{~K}$, but $3 \mathrm{DN}$ at $255 \mathrm{~K}$ (characteristic of the Arsia occurrence) correspond to approximately $1.5 \mathrm{~K}$. (2) Due to the strong dependence of the radiative cooling rate upon temperature, less shadow-induced cooling occurs when the initial temperatures are cooler. (3) The surface temperature drop during eclipse is very nonlinear with inertia, which causes less precision distinguishing between high inertia values than between low inertia values. For example, models of the Herschel occurrence yield temperature drops of $2.5 \mathrm{~K}, 1.3 \mathrm{~K}, 0.9 \mathrm{~K}, 0.7 \mathrm{~K}$, and $0.6 \mathrm{~K}$ for inertias of 84 (2), $168(4), 252(6), 336(8)$, and 420 (10), respectively.

\section{Discussion}

The results from the Arsia occurrence show that model 2 raises the derived inertias compared to the less realistic (isothermal) model 1. Adding atmospheric downward flux in model 3 reduces the derived inertias. First, we consider why the differences between the models cause these effects. Then, we consider the models' implications for the surface of Mars.

\subsection{Model Differences and Realistic Inertias}

Here we compare and evaluate our three models using the Arsia occurrence. Including the nonisothermal, initial temperature-versus-depth profile in model 2 allows the model surface to cool more quickly. For these low inertias, this dominates the simultaneous opposite (heating) effect from the additional pre-eclipse insolation caused by the inclusion of a nonzero conduction term in the surface boundary condition (see equations (3) and (5) in the appendix). Overall, the faster cooling causes higher inertias to be derived in model 2 than in model 1. 
The atmospheric downward flux added in model 3 stays constant throughout the eclipse (the validity of this assumption is discussed in the appendix), and thus keeps the total insolation higher throughout the eclipse. This causes the surface to cool more slowly. Thus, including more downward atmospheric flux causes lower inertias to be derived.

Model 3 should be the most realistic model because it includes both nonisothermal starting conditions and downward atmospheric flux. The next question is, what value of atmospheric flux in model 3 is the most realistic? This is hard to answer precisely, but fortunately the most likely possibilities make little difference in our results and do not affect our eventual scientific conclusions. The atmospheric flux depends upon the atmospheric optical depth, which is not well known for the time of the observations. Analyses of data from other Phobos ' 88 instruments have given optical depths that range from about 0.2 to 0.6 , with the most favored values somewhere near the middle of that range. According to modeling by Haberle and Jakosky [1991, Figure 4], for this range of optical depths and the local time of day of the observations, the atmospheric flux will range from $6 \%$ to $11 \%$ of the solar flux. Thus, the $20 \%$ model run shown in Figure 4 is probably an extreme and should give lower bounds on inertias. Even the 10\% run shown in Figure 5 probably gives inertias that are somewhat low. This is particularly true because the shadow was observed on a surface roughly $9 \mathrm{~km}$ (almost 1 scale height) above the $6.1 \mathrm{mbar}$ reference altitude used in the Haberle and Jakosky figure that the flux percentages came from. Atmospheric flux contributions will be reduced at higher altitudes.

The model 2 runs, which do not have any atmospheric flux contribution, should give inertia upper bounds. The real values should lie between the model 2 and model $3-20 \%$ values and are probably a little higher but not too far from the model 3-10\% values. Thus, inertias of $38(0.9)$ and 59 (1.4) probably bracket the majority of the eclipse-derived inertias in this shadow region. Ironically, because the nonisothermal effects nearly balance the atmospheric effects for these inertias, these results are nearly the same as the model 1 results (which were originally reported in Murray et al. [1991] and Betts et al. [1990]).

\subsection{Implications for the Martian Surface}

4.2.1. Arsia occurrence. The inertias derived from all the models are consistent with dust-sized particles. This is consistent with previous studies that proposed that the Tharsis region has a dust covering and is currently an area of dust deposition [Kieffer et al., 1977; Zimbelman and Kieffer, 1979; Palluconi and Kieffer, 1981; Christensen, 1986b]. It is also reasonably consistent with the low rock abundances (about 5\%) in this region [Christensen, 1982, 1983, 1986a]. Assuming a homogeneous surface, inertias of 38 to $59(0.9$ to 1.4$)$ likely imply particle sizes of approximately 5 to 10 microns $(\mu \mathrm{m})$ [Haberle and Jakosky, 1991; Kieffer et al., 1973; Jakosky, 1986]. Note, however, that these values are theoretical extrapolations from laboratory data that only go down to particle sizes of approximately $30 \mu \mathrm{m}$ [Jakosky, 1986]. Also, all of these particle sizes are for homogeneous surfaces, thus the actual surface could be composed of all 2- $\mu \mathrm{m}$ particles and an occasional rock or any number of other combinations that yield a thermally averaged surface of 5- to $10-\mu \mathrm{m}$ particles.

For the very low inertias involved in this study, diurnally derived inertias are representative of thermal skin depths of 1 or $2 \mathrm{~cm}$. Due to the short duration of the eclipse relative to the length of a Martian day, the eclipse-based inertia determinations are sensitive to thermal skin depths of only a few tenths of a millimeter. Comparison of inertias derived by the two methods can indicate the degree of layering within the upper centimeters of the surface.

We compare our eclipse inertias for this region with Viking infrared thermal mapper (IRTM)-based diurnal inertias $\left(2^{\circ} \times 2^{\circ}\right.$ bins) calculated by both Hoyashi et al. [this issue] and Palluconi and Kieffer [1981]. Hayashi et al. [this issue] used the combined surface-atmosphere model of Haberle and Jakosky [1991] along with the results of Palluconi and Kieffer [1981] and average dust opacities from Martin and Richardson [1993] in order to more accurately model the atmospheric contribution to the surface heat balance. Our eclipse inertias of 38-59 (0.9-1.4) compare to diumal inertias for the same region of 86-133 (2.1-3.2) [Hayashi et al., this issue] and 92-147 (2.2-3.5) [Palluconi and Kieffer, 1981]. Note that the atmospheric corrections of Hayashi et al. [this issue] have relatively little effect on inertias when compared with Palluconi and Kieffer [1981] for this region due to relatively high altitude and low average dust opacities. Corrections are significantly larger elsewhere on Mars [Hayashi et al., this issue].

Thus, our derived eclipse inertias are significantly lower than diumally derived inertias. However, the uncertainties in both sets of inertias make conclusions about near-surface layering tenuous. Most of the uncertainty for the Arsia occurrence eclipse inertias comes from a combination of approximating temperature drops within the eclipse (about $\pm 0.4 \mathrm{~K}$ ) and the precision of the instrument (about $0.5 \mathrm{~K}$ ). Because these errors are independent and presumably random, they combine to provide an uncertainty of approximately $\pm 0.7 \mathrm{~K}$ (which is the data uncertainty plotted in Figures 2 through 5). This translates to an inertia uncertainty of less than $\pm 12(0.3)$ for the majority of the inertias derived. It is worse for higher inertias where there is less temperature distinction between inertias, and for locations far from the shadow center. In addition, there are uncertainties due to possible inaccuracies in model inputs such as atmospheric downward flux ( $\pm 4(0.1)$, based upon trying several values), the temperature versus depth profile $( \pm 4(0.1)$, would be worse for higher inertias), and other parameters such as visible flux, flux drop with time, and exact duration of eclipse ( $\pm 8(0.2)$, based upon tests varying these parameters). Thus, the total uncertainty for the Arsia occurrence is approximately $\pm 16(0.4)$. So far, we have assumed that the uncertainty was the same, plus or minus. In reality the minus uncertainty is less than $16(0.4)$ because inertia values are more easily distinguished at lower inertias. Note also that these uncertainties are for the Arsia occurrence and will vary even for the Termoskan instrument based on time of day, surface temperature, and inertia. Therefore, future observations can be better optimized than the Termoskan observations as discussed in section 5 .

Hayashi et al. [this issue] state their uncertainty as $10-20$ $(0.2-0.5)$ for regions where albedo $\geq 0.28$ and less than $50-60$ $(1.2-1.4)$ for albedos $\leq 0.28$. The average albedo for the Arsia occurrence was 0.27 (it was 0.17 for the Herschel occurrence) [based upon Pleskot and Miner, 1981]. For the Arsia occurrence, we assume that the emissivity component of error in the Hayashi et al. [this issue] inertias is small since the average albedo of the region is nearly that of the cutoff albedo $(0.28)$, above which emissivity errors are negligible (because the emissivity does effectively equal 1 as assumed [Christensen, 1982]). However, since it is slightly below this cutoff, we include some emissivity error for comparison's sake. In this case, we have assumed that 


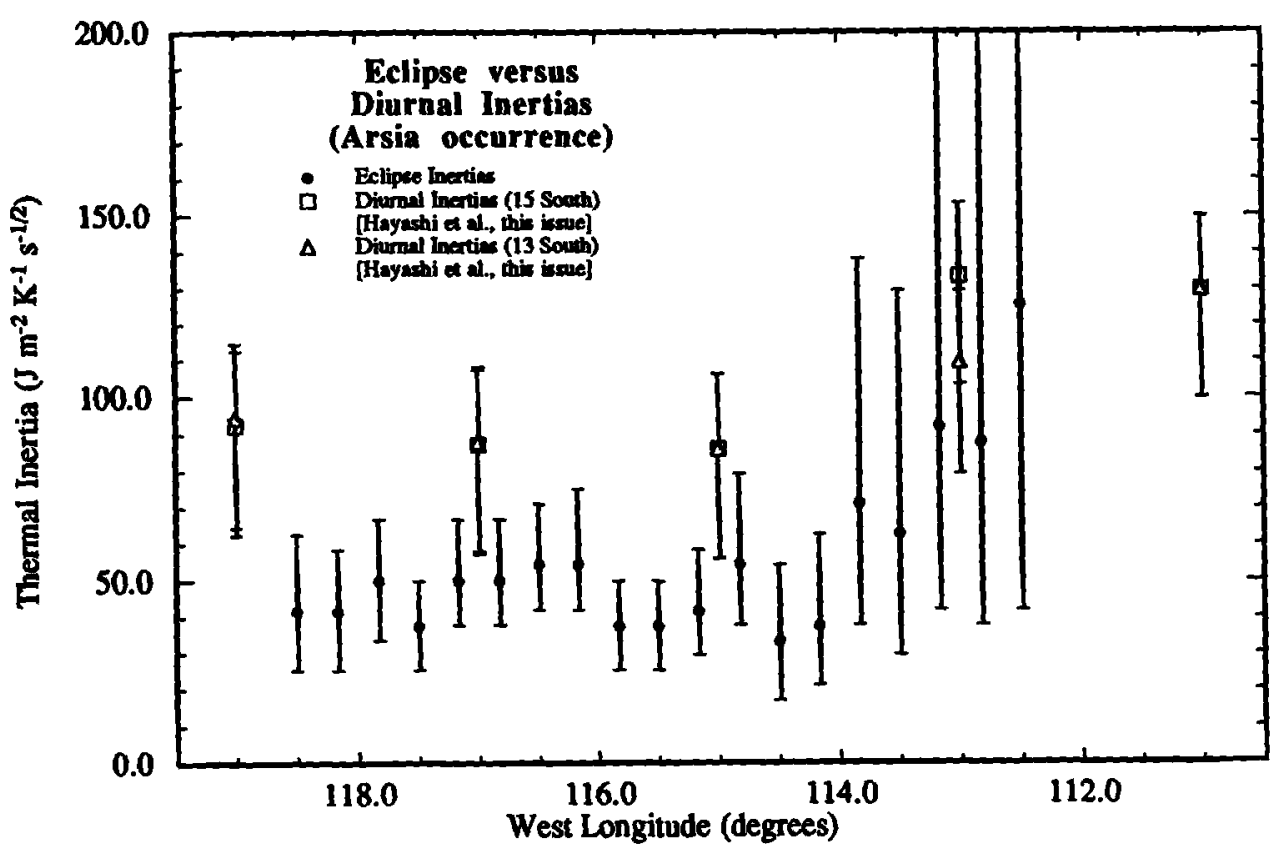

Figure 6. Eclipse versus diurnal inertias for the Arsia occurrence. Eclipse inertias from our preferred model (model 3, $A_{f}=10 \%$, see Figure 5) plotted along with the diumal inertias $\left(2^{\circ} \times 2^{\circ}\right.$ binned) of Hayashi et al. [this issue] for the same region. Note that the error bars (discussed in text) often overlap, but that the eclipse inertias are consistently lower than the diurnal inertias. Also note that both sets of inertias trend upwards towards the eastem end of the shadow. Eclipse errors also increase towards the east because the method is less sensitive far from the center of the eclipse and at higher inertias.

the emissivity error is less than $10(0.2)$ in the Hayashi et al. [this issue] diurnal inertias for the Arsia occurrence region.

Figure 6 compares eclipse inertias (with uncertainties found independently for each point) with the Hayashi et al. [this issue] inertias, which are plotted with uncertainties of +20 and -30 (emissivity error will only act in the negative direction since it causes the inertias to be overestimated). Note that the eclipse inertias are, as discussed, consistently lower than the diumal inertias, but that error bars often overlap. Also, notice that the eclipse inertias trend upwards towards the east as do the diurnal inertias. Unfortunately, the eclipse errors become large in that region because the eclipse is ending and the inertias are higher. However, the trend appears in the last few eclipse inertia points, making the simultaneous upward trend of both eclipse and diumal inertias more believable than the error bars from any one point would indicate.

Thus, due to the uncertainties in both our eclipse inertias and in diumally derived inertias, we are unable to say for certain that there is layering in the Arsia occurrence region. If there is, we can say that it is mostly fine dust over mostly less fine dust. The easiest and most interesting locations to look for layering with future missions will be in regions that have higher diurnal inertias (indicative of average particle sizes significantly larger than dust) than those of the Arsia occurrence.

The few tenths of a millimeter sampled by eclipse cooling measurements should present a fair representation of the surface that is sensed by optical and near-infrared instruments. Thus, for the region studied, it is unlikely that optical and near-infrared instruments would sample any significant amount of bare (not covered by dust) rock surfaces.

4.2.2. Herschel occurrence. The inertia lower bound of 80 (2) that we derive for the Herschel occurrence is consistent with diumal inertias for the shadowed region: 198-252 (4.7-6.0) [Hayashi et al., this issue] and 239-301 (5.7-7.2) [Palluconi and Kieffer, 1981]. These diumal inertias are consistent with 8 homogeneous surface of sand-sized particles. Unfortunately, because we are only able to derive a lower bound for this occurrence, and because that lower bound is so low, we are unable to derive any strong physical conclusions about the surface. If eclipse inertias are actually as low as 80 (2), then one could say there is a dust covering, but since they also could be significantly larger than 80 (2), the surface may be totally devoid of layering. Note, that if more advantageous observations (midday, greater sensitivity, etc.) are obtained by future spacecraft, the question of layering can be addressed effectively by the shadow analysis method.

\subsection{Summary}

Our analyses have shown that thermal and visible observations of the shadow of Phobos can be used to derive physical information about the upper millimeters of the Martian surface. Combining our preferred eclipse model with the Termoskan thermal data implies inertias for the Arsia Mons shadow occurrence that mostly fall within the range 38 to $59(0.9$ to 1.4). These inertias correspond to dust-sized particles ( 5 to 10 $\mu \mathrm{m}$ for a homogeneous surface based upon theoretical extrapolations of laboratory data). The presence of dust at the surface is consistent with previous theories of Tharsis as a current area of dust deposition. Thus, most of the upper couple centimeters is likely composed of unbonded, few micron dust particles. There may be a slight degree of layering, and the eclipse inertias and diurnal inertias both appear to increase towards the eastern end of the shadow. However, uncertainties 
make these last two conclusions tenuous. Analysis of a shadow occurrence near the crater Herschel places a lower bound on eclipse inertia of 80 (2).

These findings obviously do not preclude layering or higher inertias elsewhere. Questions of geographic variability of the upper millimeter of the surface can be addressed to some extent in the future using the two other Termoskan observed occurrences of the Phobos shadow, but can best be addressed by future missions with optimized observations.

\section{Future Phobos Shadow Research}

Our results indicate that analyses of Martian surface cooling, due to the passage of the shadow of Phobos, can yield unique and interesting results about the current physical state of the upper millimeters of the Martian surface. However, the fortuitous Termoskan observations of the shadow are very limited in geographic coverage and, with the exception of the Arsia occurrence, are very limited in accuracy. Because of these limitations, some of the key questions that could be addressed by this type of observation remain unanswered, e.g., is there a dust layer of at least millimeters in thickness covering much of Mars?, or are dust layers regional in nature? Thus, we recommend that observations of the shadow be attempted with future missions. Future observations should improve upon the Termoskan data in geographic coverage, accuracy of the observations, and temporal coverage.

Ideal characteristics of future observations would include targeting midday shadow passages to maximize the pre-eclipse temperature, which will maximize the shadow temperature drops, the distinction between different inertias during eclipse, and the signal to noise ratio; utilizing framing rather than (Termoskan-like) scanning imaging systems so the entire image is taken simultaneously, and taking several images as the shadow crosses the surface so that pre-eclipse, eclipse, and posteclipse temperatures are more accurately known for any given location; and having at least one broad band thermal channel and one broad band visible channel.

Observations will need to be taken during periods surrounding the equinoxes when the shadow will cross the Mars surface. The least shadow distortion and atmospheric interaction will occur in the equatorial region at equinox and during periods near that time. Because Phobos has an orbital period of approximately 8 hours, there should be ample opportunity to observe several shadow crossings at several latitudes. The most advantageous orbits for the observations will be equatorial (as is Phobos' orbit) so that the shadow can be followed across the surface and so preeclipse and posteclipse observations can easily be obtained. One ideal observing situation would be to have the instruments on a lander on the Mars facing side of Phobos. Then, observations could be taken merely by pointing in an antisolar direction. They will be particularly useful if the field of view is wide enough and if observations are taken frequently enough so a number of locations are observed, with temporal coverage for each location providing pre-eclipse, eclipse, and posteclipse temperatures.

\section{Appendix: Thermal Models of the Eclipse Cooling}

In order to derive thermal inertias from the eclipse observations, we modeled the cooling of the surface for several thermal inertias and compared the results to the observed cooling. We present here descriptions and results from three different, but related, models: model 1 , the isothermal model, which assumes that initially all depths are at the same temperature; model 2, the nonisothermal model, which utilizes an initial temperature with depth profile derived from the Clifford et al. [1987] diurnal thermal model; and model 3, the nonisothermal atmospheric model, which adds a downward atmospheric flux term to model 2 . All three models are presented here for comparison and completeness. Model 1 was used to produce the results in Murray et al. [1991]. Model 2 improves on model 1 . Model 3 is the most complete model because it includes atmospheric re-radiation; however, the amount of atmospheric flux is highly dependent upon poorly known atmospheric conditions at the time of the observations. Thus, it is useful to consider model 2 separately from model 3.

All of the models solve the heat equation:

$$
\frac{\partial T}{\partial t}=\frac{1}{\rho c} \frac{\partial}{\partial z}\left(k \frac{\partial T}{\partial z}\right)
$$

where $T$ is temperature, $t$ is time, $\rho$ is density, $c$ is specific heat, $k$ is thermal conductivity, and $z$ is depth. This equation is solved using the same numerical method used by Clifford et al. [1987]. This is essentially the same diumal model described by Kieffer et al. [1977]. Modeling the eclipse rather than diumal and seasonal temperature variations requires several important differences between the eclipse models and the overall Clifford et al. model. Parameters such as the timescales used and inputs such as the decrease in visible flux with time in the eclipse must be changed. Where possible, we use the actual Termoskan data to make these changes, rather than relying on theoretical calculations. For example, we use the Termoskan visible data to derive the decrease in absorbed solar flux with time. Using the data where possible decreases the potential error introduced by poorly known factors such as the complex geometry including spacecraft rocking, atmospheric effects, and uncertainties in albedos.

All of the models produce model temperatures as a function of time in eclipse for a given value of thermal inertia. Actual thermal inertias are estimated by first running a model for several values of thermal inertia; then, model temperatures are compared with Termoskan data temperatures to find inertias for given values of time eclipsed. This comparison was discussed in section 3.

In the rest of this section we describe the three models by pointing out their differences from the Clifford et al. [1987] and Kieffer et al. [1977] models. In the first five points below, we present the elements that apply to all three models. Then, in the last two points, we address the differences in the three models. Hence, the following are the eclipse model features that are different from the Clifford et al. [1987] and, by analogy, the Kieffer et al. [1977] model.

1. Timescales (depth scales). We have adjusted all timerelated variables to account for the 23-s eclipse timescale, rather than diumal timescales. The iteration time, along with the modeled inertia, determines the thickness and depths of the model compartments based upon finite difference stability criteria [e.g., Clifford et al., 1987]. We changed the interval between iterations from several minutes to $0.001 \mathrm{~s}$. Models with smaller iteration times were tested but showed no appreciable difference from the models run with $0.001-\mathrm{s}$ iterations.

2. Use of orbital information and albedos. Neither Mars orbital information nor albedo information is used in the eclipse models. How these are avoided will become more clear in the specific discussions of elements below. We generalize this point here to emphasize the major differences with the Clifford et al. 
[1987] model. Note that although the eclipse models do not use orbital information or albedo information directly, models 2 and 3 do use them indirectly because we ran the Clifford et al. [1987] model to generate the initial temperature with depth profiles (discussed in point 6 below). Errors in albedo introduce only very minor errors when used in this indirect fashion.

3. Downward atmospheric flux term. The downward atmospheric flux model term is set equal to 0 rather than $2 \%$ of the noontime solar insolation. Models 1 and 2 have no downward atmospheric flux and model 3 incorporates it elsewhere within the model.

4. Initial surface temperature. Rather than calculating this from orbital and albedo considerations, the initial (pre-eclipse) surface temperature is assigned a value that is a representative of observed Termoskan temperatures for regions just outside the shadow (e.g., $255 \mathrm{~K}$ for the Arsia Mons occurrence).

5. Relative insolation as a function of time. In order to model the cooling as a function of time eclipsed, the model needs a description of the total absorbed insolation as a function of time within the eclipse. For models 1 and 2 this is equivalent to the absorbed solar insolation as a function of time. For model 3 this includes atmospheric as well as solar insolation as discussed more fully in point 7 below. We derive the shape of the insolation-versus-time function from the visible data and the magnitude from the thermal data. In point 7 below we consider the magnitude of the pre-eclipse insolation used, which differs for each of the three models.

Here we discuss the shape of the insolation versus time function. We use this same relative insolation function in all three models. To find the relative insolation, we did the following: A - calculated the duration of the eclipse for a point on the surface; B - derived a function that connects location within the shadow in the data to the amount of time that location has been in eclipse; $\mathrm{C}$ - used the visible channel eclipse profile to fit the decrease in absorbed solar insolation as a function of location within the shadow, and D - combined B and C to derive relative insolation as a function of time in eclipse. These steps are accomplished in the following manner.

A. Combining the maximum width of a north-south profile, the orbital speed of Phobos, and the rotational speed of Mars gives an eclipse duration of just under $23 \mathrm{~s}$.

B. We assumed the E-W rocking motion to be uniform over the brief Arsia Mons shadow observation. This assumption is justified both by the short duration of the observation and by the smoothness and shape of the E-W visible shadow profile. Thus, we were able to develop a linear relation between the number of lines into eclipse and the time a location had been in eclipse using the observed E-W size of the shadow (approximately 180 lines for the Arsia occurrence) and the eclipse duration calculated above (approximately $22.6 \mathrm{~s}$ ). So, for example, the 90 th line after the beginning of eclipse had been in some form of eclipse for approximately $11.3 \mathrm{~s}$.

C. The next step was to determine the relative decrease in solar insolation as a function of location within the shadow. We assumed that the visible channel signal was linearly proportional to visible flux, consistent with the visible detector's characteristics and with all approximate flux calibrations of the visible channel [Betts, 1993]. Thus, the E-W visible channel signal profile should approximate the visible flux decrease within the shadow. We used this profile to quadradically fit the relative decrease in solar insolation as a function of location within the shadow. Note that in the darkest part of the shadow the flux decreased by approximately $30 \%$. The advantage of using the visible channel profile instead of theoretical calculations of the flux decrease is that it already incorporates atmospheric effects as well as geometric distortion due to the spacecraft rocking. Position within the visible channel can be directly correlated with position within the thermal channel to within approximately 1 pixel [Betts, 1993; 1992].

D. Combining $\mathbf{B}$ and $\mathbf{C}$ gives the relative absorbed solar insolation as a function of time eclipsed, $f(t)$. This function can vary from 0 to 1 , where 1 represents the pre-eclipse insolation.

The only remaining step for modeling the insolation is to get an absolute pre-eclipse absorbed solar insolation that this relative function can be mated with. This is discussed separately below in point 7. First, we discuss the initial temperature with depth profile used because that will be important for point 7 .

6. Initial temperature with depth profile. Model 1 is isothermal before the onset of eclipse with all depths set equal to the pre-eclipse representative surface temperature (255 $\mathrm{K}$ for the Arsia occurrence). Models 2 and 3 utilize more realistic nonisothermal subsurface pre-eclipse temperature profiles. As mentioned, establishing a reasonable estimate for this temperature with depth profile is the only place in the models where either albedo or Mars orbit information is used. Even here, they are used indirectly. Before each run of either models 2 or 3, we ran our adaptation of the Clifford et al. [1987] diurnal model utilizing the appropriate season $\left(L_{s}=18^{\circ}\right)$, approximate latitude $\left(14^{\circ} \mathrm{S}\right)$, and approximate albedo $(0.27$ for the Arsia occurrence and 0.17 for the Herschel occurrence as taken from the $1^{\circ} \times 1^{\circ}$ binned bolometric albedos of Pleskot and Miner [1981]). Using the diumal model output corresponding to the local time of day of the eclipse, about $10 \mathrm{H}$ for the Arsia occurrence (where $24 \mathrm{H}=1$ Martian day), we estimated the preeclipse temperature as a function of depth with a linear approximation:

$$
T_{j}=T_{i}-\frac{D_{j}\left(T_{s d}-T_{j d}\right)}{D}
$$

where $T_{j}$ is the temperature of the $j$ th compartment, $T_{i}$ is the initial surface temperature, i.e., $255 \mathrm{~K}$ for the Arsia occurrence, $D_{j}$ is the depth of the top of the $j$ th eclipse depth compartment, $T_{s d}$ is the surface temperature in the diurnal model, $T_{f d}$ is the temperature of the first depth compartment in the diurnal model, and $D$ is the depth of the first depth compartment in the thermal model. Note that this pre-eclipse temperature with depth profile depends upon the thermal inertia used in the diurnal model. Thus, for each value of inertia nun in the eclipse model, we first modeled the depth profile using that inertia in the Clifford et al. [1987] diumal model. This gave new values of $T_{s d}, T_{f d}$ and $D$ to use in equation (2) and the eclipse model. Then, for a given inertia, all values of $T_{j}$ could be found as a linear function of $D_{j}$.

7. Pre-eclipse absorbed insolation. Finally, we need the value of the pre-eclipse absorbed insolation to use with the relative function found in part 5 above. To do this, we use the surface equilibrium boundary condition discussed by Kieffer et al. [1977] and elsewhere:

$$
\frac{S(1-A) \cos i}{R^{2}}+F_{a}=\varepsilon \sigma T^{4}-k \frac{d T}{d z}-L \frac{d M}{d t}
$$

where $S$ is the solar constant, $A$ is the bolometric Bond albedo, $i$ is the solar incidence angle, $R$ is the Mars heliocentric distance, $F_{a}$ is the downward atmospheric flux, $\varepsilon$ is the surface emissivity, $\sigma$ is the Stefan-Boltzmann constant, $T$ is the surface temperature, $k$ is the thermal conductivity, $z$ is the depth, $d T / d z$ is the change 
in temperature with depth evaluated at the surface, $L$ is the $\mathrm{CO}_{2}$ latent heat of condensation, and $M$ is the mass per unit area of $\mathrm{CO}_{2}$ frost.

The terms on the left in equation (3) represent the total absorbed insolation, i.e., what we need to know for pre-eclipse conditions. The first term on the left is the absorbed solar insolation. The second term on the left represents the downward infrared atmospheric flux that reaches the surface. This thermal infrared flux is assumed to be absorbed completely by the surface. The first term on the right is the surface emission. Here we assume the emissivity, $\varepsilon$, to be 1 , as was done by Kieffer et al. [1977]. The second term on the right is the surface conduction term. The third term on the right is the term representing latent heat from carbon dioxide sublimation or condensation. The temperatures involved in this shadow analysis are far above the carbon dioxide condensation temperature, so this term is set to zero.

The three models differ in which terms are ignored in calculating the total absorbed insolation. Model 3 only ignores the $\mathrm{CO}_{2}$ latent heat term. Model 2 also ignores the atmospheric term, and model 1 additionally ignores the conduction terms.

In principle, the total absorbed insolation could be calculated directly in any of these models from the terms on the left side of equation (3). Instead, we choose to solve for the total insolation by finding the terms on the right side. There are three reasons to do this. One, by more explicitly using the Termoskan data, we avoid uncertainties in albedo, and the orbital geometry, and we significantly reduce the effects of atmospheric scattering. Two, utilizing the same initial surface temperature, $255 \mathrm{~K}$, that is already used in the pre-eclipse temperature profile will result in greater initial consistency within the model. Three, in model 3, to calculate the terms on the left side, we also would need to directly calculate the atmospheric downward flux. This is very difficult to do accurately due to the poor knowledge of the state of the Martian atmosphere at the time of the observations. By using the surface emission and conduction terms to determine the total insolation, we avoid these problems.

We summarize the differences between our three eclipse models in Table 2. Here we describe in detail the differences in the insolations used. In model 1 , which ignores the atmospheric, conduction, and latent heat terms, the pre-eclipse absorbed insolation, $I_{0}$, is approximated by $\sigma T^{4}$, where $\sigma$ is the StefanBoltzmann constant and $T$ is a representative value of the surface temperature derived from the data itself ( $255 \mathrm{~K}$ for the Arsia occurrence).

Noneclipsed surface temperatures, and the corresponding noneclipsed absorbed insolations, will change over the region covered by the eclipse and differ from the representative surface temperature ( $255 \mathrm{~K}$ for Arsia); however, the short duration and small area covered by the Arsia eclipse keep these variations small. Also, as discussed in section 3, we use temperature drops within the eclipse rather than absolute temperatures for comparison with the model to minimize errors caused by insolation or initial temperature errors in the model.

Ignoring the conduction term, $k(d T / d z)$, in model 1 is consistent with the initial isothermal assumption in this model which implies $d T / d z=0$. The insolation as a function of time, $I(t)$, combines $I_{0}$ with the relative flux decrease as a function of eclipsed time, $f(t)$, that was derived from the visible channel data in step 5 . Thus, for model 1 ,

$$
I(t)=I_{0} f(t)
$$

Model 2 differs from model 1 by approximating the pre-
Table 2. Summary of Eclipse Model Differences

\begin{tabular}{ccccc}
\hline Model & $\begin{array}{c}\text { Pre-eclipse } \\
\text { Temperature } \\
\text { With Depth }\end{array}$ & $\begin{array}{c}\text { Includes } \\
\text { Atmospheric } \\
\text { Flux }\end{array}$ & $I_{0}$ & $I(t)$ \\
\hline 1 & Isothermal & No & $\sigma T^{4}$ & $I_{s} f(t)$ \\
2 & Nonisothermal & No & $\sigma T^{4}-k(d T / d z)$ & $I_{s} f(t)$ \\
3 & Nonisothermal & Yes & $\sigma T^{4}-k(d T / d t)$ & $I_{a}+I_{s} f(t)$
\end{tabular}

I0, pre-eclipse total absorbed insolation $=I_{s}$ when there is no atmospheric downward flux, $I(t)$, total insolation as a function of time in eclipse; $I_{s}$, pre-eclipse absorbed solar insolation; $I_{a}$, absorbed atmospheric insolation; $f(t)$, relative absorbed solar insolation (on a scale of 0 to 1 ) as a function of time in eclipse; $t$, time.

eclipse absorbed insolation, $I_{0}$, by

$$
I_{0}=\sigma T^{4}-k \frac{d T}{d z}
$$

Note that including the conduction term here is consistent with the nonisothermal initial conditions of this model. After the initial temperature profile with depth is derived for this model as described in step 6 above, $d T / d z$ is approximated by Taylor expansions of the first three depth steps and their temperatures [Clifford et al., 1987]. Note that for the midmorning Arsia eclipse observation, $d T / d z$ is negative. Thus, $I_{0}$ will be larger in model 2 than in model 1 . The total insolation as a function of time, $I(t)$, is found in model 2 in the same way as in model 1 , using equation (4).

Model 3 determines the value of $I_{0}$ in the same manner as model 2, using equation (5). Model 3 differs by assuming $I_{0}$ to be composed of an atmospheric component (downward infrared flux) in addition to the solar component. Thus, model 3 differs from model 2 in the calculation of $I(t)$. The relative flux drop as a function of time derived in step 5 is based upon the visible channel, which sensed only the solar flux drop. We assume that the absorbed downward atmospheric flux, $I_{a}$, remains constant throughout the eclipse. This assumption is very reasonable because (1) the shadowed portion of the atmosphere represents less than approximately $0.5 \%$ of the volume of atmosphere that contributes infrared atmospheric flux to the shadowed surface; and (2) the flux from the shadowed atmosphere will only drop on the order of $10-20 \%$ even assuming very large amounts of atmospheric cooling, so the total atmospheric flux variation will be less than $0.1 \%$. Thus, in our model, $I_{a}$ remains constant throughout the eclipse, whereas the absorbed solar insolation, $I_{s}$, will vary with eclipse time as described by the function $f(t)$ that was derived in step 5 . Thus, rather than using equation (4) as models 1 and 2 did, model 3 uses

$$
I(t)=I_{a}+I_{s} f(t)
$$

Here, $I_{0}$, which is what we derived from the surface emission and conduction terms in equation (5), represents the combination of $I_{a}$ and $I_{s}$ i.e.,

$$
I_{0}=I_{s}+I_{a}
$$

$I_{a}$ will depend strongly upon the amount of dust in the atmosphere as well as to some extent the elevation of the surface. In order to conveniently model different values of $I_{a}$, we define $I_{a}$ 
as a fraction, $A_{f s}$ of $I_{s}$. Thus,

$$
I_{a}=A_{f} I_{s}
$$

Combining equations (6), (7), and (8) gives an equation dependent upon only $I_{0}$ and $A_{f}$ :

$$
I(t)=\frac{\dot{I}_{0}\left(f(t)+A_{f}\right)}{1+A_{f}}
$$

which reduces to equation (4) when $A_{f}=0$. So, using equation (9), the model can be run for several values of $A_{f}$ to determine the effects of different amounts of downward atmospheric flux.

Acknowledgments. We thank Ken Herkenhoff and Ken Edgett for their helpful and detailed reviews, Doug Nash, Dewey Muhleman, and Andy Ingersoll for helpful comments on the manuscript, Joan Hayashi for providing digital versions of the Hayashi et al. [this issue] inertias; and Brice Jakosky, David Paige, and Hugh Kieffer for insightful discussions. We also thank A Selivanov, M. Naraeva, V. Kharlamov, and Y. Gektin for assistance with the data, and R. Eby, R. McLendon, K. Hudelson, and S. Welch for assistance with tasks at SJ. Funding for this research was provided by NASA grants NAGW-1426 and NAGW-2491. Division of Geological and Planetary Sciences, California Institute of Technology contribution 5384.

\section{References}

Betts, B. H., Edited Termoskan and raw data files and descriptions, on the PDS (Planetary Data System) Phobos '88 CD-ROM, vol. PHB -1001, May 1992.

Betts, B. H., Thermal and visible studies of Mars using the Termoskan data set, Ph.D. thesis, Calif. Inst. of Technol., Pasadena, 1993.

Betts, B. H., T. Svitek, M. L. Santee, B. C. Murray, D. Crisp, D. A Paige, M. Naraeva, and A. Selivanov, Preliminary quantitative assessment and analysis of Phobos 88 Termoskan observations of Mars (abstract), Lumar Planet. Sci., XXI, 77-78, 1990.

Christensen, P. R, Martian dust mantling and surface composition: Interpretation of thermophysical properties, J. Geophys. Res., 87, 9985-9998, 1982.

Christensen, P. R., The distribution of rocks on Mars (abstract), Lumar Planet. Sci., XIV, 109-110, 1983.

Christensen, P. R., The spatial distribution of rocks on Mars, Icarns, 68, 217-238, 1986a.

Christensen, P. R., Regional dust deposits on Mars: Physical properties and global distribution, J. Geophys. Res., 91, 3534-3546, 1986b.

Clifford, S. M., C. J. Bartels, and E. P. Rubenstein, The Mars thermal model (MARSTHERM): A FORTRAN 77 finite-difference program designed for general distribution, Luniar and Planetary Institute, Houston, Tex, 1987.

Haberle, R. M., and B. M. Jakosky, Atmospheric effects on the remote determination of thermal inertia on Mars, Icarus, 90, 187-204, 1991.

Hayashi, J. N., B. M. Jakosky, and R. M. Haberle, Atmospheric effects on the mapping of Martian thermal inertia and thermally derived albedo, J. Geophys. Res., this issue.

Jakosky, B. M., On the thermal properties of the Martian fines, Icarus, 99, 117-124, 1986.

Kieffer, H. H., S. C. Chase Jr., E. Miner, G. Münch, and G. Neugebauer, Preliminary report on infrared radiometric measurements from the Mariner 9 spacecraft, J. Geophys. Res., 78, 4291-4312, 1973.

Kieffer, H. H., T. Z. Martin, A R. Peterfreund, B. M. Jakosky, E. D. Miner, and F. D. Palluconi, Thermal and albedo mapping of Mars during the Viking primary mission, J. Geophys. Res., 82, 4249 . 4291, 1977.

Martin, T. Z. and M. I. Richardson, New dust opacity mapping from Viking Infrared Thermal Mapper Data, J. Geophys. Res., 98, 10941-10949, 1993.

Murray, B. C., et. al, Preliminary assessment of Termoskan observations of Mars, Planet. Space Sci., 39(1/2), 237-265, 1991.

Palluconi, F. D., and H. H. Kieffer, Thermal inertia mapping of Mars from $60^{\circ} \mathrm{S}$ to $60^{\circ} \mathrm{N}$, Icards, 45, 415-426, 1981.

Pleskot, L. K., and E. D. Miner, Time variability of Martian bolometric albedo, Icarus, 45, 179-201, 1981.

Selivanov, A. S., M. K. Naraeva, A S. Panfilov, Yu. M. Gektin, V. D. Kharlamov, A V. Romanov, D. A Fomin, and Ya. Ya. Miroshnichenko, Thermal imaging of the surface of Mars, Nature, 341, 593-595, 1989.

Zimbelman, J. R, and H. H. Kieffer, Thermal mapping of the northern equatorial and temperate latitudes of Mars, J. Geophys. Res., 84, 8239-8251, 1979.

B. H. Betts., San Juan Capistrano Research Institute, 31872 Camino Capistrano, San Juan Capistrano, CA 92675. (e-mail: betts@sji.org)

B. C. Murray, Division of Geological and Planetary Sciences, California Institute of Technology, Pasadena, CA 91125. (e-mail: bcm@earthl.gps.caltech.edu)

T. Svitek, Orbital Sciences Corporation, 21700 Atlantic Blvd. Dulles, VA 20166.

(Received March 1, 1994; revised January 13, 1995; accepted January 13, 1995.) 